\title{
Konten und Codes
}

\author{
E. Taverna
}

Codes ordnen und verknüpfen. Sie übersetzen die chiffrierte Botschaft in den Klartext, sie stehen für Sprachregeln, Kleidernormen und Verhaltensregeln. Die digitalen Codes verändern zunehmend das alphabetische Denken, das ein Denken in Bildern ist.

\section{Kodierungen ...}

Medizinische Kodierungen erfordern Spezialisten, die immer häufiger in Inseraten der Schweizerischen Ärztezeitung gesucht werden. Sie übertragen die Informationen einer Sprache, die in diesem Fall bereits eine codierte Fachsprache ist, in eine numerische Struktur, die, elektronisch verarbeitet, statistische Aussagen erlaubt. Ärzte meiner Generation haben sich im allgemeinen bisher wenig für diese Arbeit interessiert, obwohl auch vor dem PC Zahlensysteme, mit und ohne Lochkarten, als Hilfsmittel verwendet wurden. Jedes Tarifsystem hat schon lange vor TARMED die Sprechstunde in eine Zahlenkolonne verwandelt. Wir müssten also bestens auf eine Epoche vorbereitet sein, in der alle Lebensbereiche digitalisiert werden.

Eine neue Spezialistengruppe brütet über Dossieranalysen nach Kodierregeln des BFS und der Schweizerischen Expertengruppe für Klassifikationen, die im Sinne der OECD-Methodik die Geldströme auf Gesundheitskonten aufteilen. Diese Konten müssen folgende Fragen beantworten: 1. Woher kommt das Geld? (Finanzierungsträger); 2 . Wer erhält das Geld? (Leistungserbringer); 3. Welche Leistungen werden produziert und welche Güter gekauft? Die Unterteilung der Patientenpopulation nach 641 Pathologiegruppen, Haupt- und Nebendiagnosen ermöglicht, zusammen mit Tageskosten und Abteilungspauschalen, die ersten «pathologiebasierten Finanzierungen». Gemäss dem Code-Info Nr. 10 [1] haben gut kodierte Diagnosen und Behandlungen für Spitäler eine «strategische Bedeutung» erlangt, weil Fehler finanziellen Folgen haben. Kein Wunder, dass aus Teilzeitstellen gut dotierte Vollzeitstellen werden, die im Rahmen der neuen Arithmetik mit den 4. und 5. Stellen der ICD-Systematik vertraut sind. In der neuen Fachsprache gemäss Plausibilisierungskonzept Version 2.5: «Die Angabe von dreistelligen Schlüsselnummern bewirkt eine Fehlermeldung, falls in der ICD-10 unter der gegebenen Kategorie vierstellige Unterteilungen bestehen.» Die rasch sich entwickelnde Programmiersprache erfordert, mehr noch als medizinische, solide Informatikkenntnisse und eine minutiöse Umsetzung medizinischer Leistungen in die neuen Codes. Ein Facharzttitel FMH für Medizinische Informatik wird nicht lange auf sich warten lassen.

\section{... und Nebenwirkungen}

Im immer engmaschigeren Zahlenkäfig der generierten Daten werden Machtpositionen definiert, weil gemäss Dr. med. M. Denz [2] die Kodierungsautonomie zugleich eine Frage der beruflichen Selbstbestimmung sei. Die berufliche Identität setzt offenbar eigene Datenerhebungen und Klassifikationen voraus. Die Grenzen der neuen Reiche im Gesundheitswesen werden durch Kodierungshoheiten abgesteckt.

Wer die Definitionsmacht hat, wird dafür sorgen, dass die Zahlen seine Absichten «beweisen», ganz nach dem Motto: «Traue nur der Statistik, die du selber gefälscht hast.» Doch auch das wäre nur die Fortsetzung alter Gefechte mit neuer Munition.

Die neuen Werkzeuge der Statistik zielen auf ein umfassendes, kohärentes und international vergleichbares System, das mit anderen Statistiken des Wirtschafts- und Sozialbereiches kompatibel sein soll. Die Verknüpfung dieser gewaltigen Datenmengen ist nur digital möglich. Die Konsequenzen aus der Sicht des Datenschutzes sind unabsehbar. Unabsehbar sind auch die Folgen für unser Denken, in diesem Fall für das medizinische Denken.

Kritiker der Digitalisierung zweifeln daran, dass unser altes lineares Denken mit seinen linearen Rechtschreiberegeln mit der neuen Raum- und Zeiterfahrung Schritt halten kann. Denn wie sich das aufs Alphabet gestützte Denken gegen Magie und Mythos engagiert habe, so werde das neue, auf digitale Codes gestützte Denken das Alphabet und mit ihm auch eine ganze Kultur verdrängen. Alles Neue macht Angst. Ob die Mathematiker die Schriftsteller verschlucken ist noch offen. Die gegenwärtige Medizinkritik ist voll von diesen Widersprüchen. Einerseits wird das Individuelle und Einmalige einer Krankheit oder Behinderung mit Patienten- 
rechten in einem Masse betont, dass es bald nicht mehr darum zu gehen scheint, was in einem bestimmten Fall gemacht wird, sondern viel mehr darum, wer darüber entscheidet, der Patient oder der Therapeut. Auf der anderen Seite provozieren die steigenden Kosten ein Kontrollsystem, das Patientendaten in einem Mass verallgemeinert, wie es nie zuvor möglich war. Immer neue Klassifikationen, die zum Beispiel den Gesundheitszustand der Bevölkerung konkret beschreiben wollen, dienen als ökonomische und politische Entscheidungsgrundlagen. Neue Schemas ergänzen die ICD-10 um weitere Evaluationsbereiche wie Körperfunktionen, Körperstrukturen, Aktivitäts- und Partizipationsbereiche sowie Kontextfaktoren. Was als Instrument für die Evaluation von Behinderungen ent- wickelt vorliegt, wird mit Sicherheit, um weitere Faktoren ergänzt, auf die ganze Bevölkerung ausgedehnt. Der neue Schweizer Pass, der Raum für biometrische Angaben reserviert, gibt die Richtung an. Mehr denn je spiegeln die medizinischen Anwendungen den gesellschaftlichen Trend.

\section{Literatur}

1 Code-Info 2/01, Die Zeitschrift der Kodierung, Nummer 10, Dezember 2001.

2 Denz MD. Medizinische Kodierung ist weit mehr als ein lästiges Übel! Schweiz Ärztezeitung 2002;83(8):375-8.

- Kosten des Gesundheitswesens. Ergebnisse und Entwicklungen 1995-1999. BfS-Heft 14, Bestellnummer 026-9900, Tel. 0327136060. 